\title{
OPEN Why do nanowires grow with their c-axis vertically-aligned in the absence of epitaxy?
}

\begin{abstract}
Almog R. Azulay ${ }^{1}$, Yury Turkulets ${ }^{1}$, Davide Del Gaudio ${ }^{2}$, R. S. Goldman ${ }^{2}$ \& Ilan Shalish ${ }^{1 *}$
Images of uniform and upright nanowires are fascinating, but often, they are quite puzzling, when the substrate is clearly not an epitaxial template. Here, we reveal the physics underlying one such hidden growth guidance mechanism through a specific example - the case of $\mathrm{ZnO}$ nanowires grown on silicon oxide. We show how electric fields exerted by the insulating substrate may be manipulated through the surface charge to define the orientation and polarity of the nanowires. Surface charge is ubiquitous on the surfaces of semiconductors and insulators, and as a result, substrate electric fields need always be considered. Our results suggest a new concept, according to which the growth of wurtzite semiconductors may often be described as a process of electric-charge-induced self-assembly, wherein the internal built-in field in the polar material tends to align in parallel to an external field exerted by the substrate to minimize the interfacial energy of the system.
\end{abstract}

Nanowires often adopt specific orientation during their growth despite the lack of eptitaxial guidance from the substrate. While this phenomenon may not be limited to nanowires, it has become more easily apparent with nanowires, with most of the evidence coming from $\mathrm{ZnO}$. Several unique features make $\mathrm{ZnO}$ one of the most intensively studied semiconductors today ${ }^{1}$. To date, $\mathrm{ZnO}$ has been used as a transparent conductor in photovoltaic applications $s^{2}$, varistor for voltage surge protection ${ }^{3}$, solar blind photodetector ${ }^{4}$, gas sensor ${ }^{5}$, and photocatalyst material ${ }^{6}$. It has also been proposed for several future applications, as transparent field effect transistors ${ }^{7}$, UV light-emitting diodes ${ }^{8}$, memristors ${ }^{9}$, biosensors ${ }^{10}$, and spintronic devices ${ }^{11,12}$. Growth of $\mathrm{ZnO}$ on $\mathrm{Si}$ is vital for integrating this material into the present microelectronic technology. One peculiar observation that has become more easily apparent with the growth of $\mathrm{ZnO}$ nanowires is that on substrates such as glass and silicon, that do not provide an epitaxial template, $\mathrm{ZnO}$ grows in a direction along the $\mathrm{c}$ axis, i.e. it is preferentially c-oriented ${ }^{13}$. For example, in Fig. 1a, the presence of $3 \mathrm{ZnO}$ hexagonally-shaped mesas on a $\mathrm{Si}(100)$ substrate confirms that the mesas are indeed c-oriented.

The sides of the hexagonal mesas are not mutually parallel, as shown schematically in Fig. 1b, thus confirming the lack of epitaxial relations with the substrate. Growth of c-oriented nanowires on Si substrate, as shown in Fig. 1c, has been reported in several papers. For example, c-oriented $\mathrm{ZnO}$ nanowire arrays have been grown on silicon or glass substrates without the use of a preexisting textured thin film. These were synthesized at temperatures ranging from 400 to $600^{\circ} \mathrm{C}$ using metal organic vapor phase epitaxy ${ }^{14}$, chemical vapor deposition (CVD $)^{15}$, atomic layer deposition ${ }^{16}$, chemical vapor transport ${ }^{17}$, or a hydrothermal approach ${ }^{18,19}$. In many cases, the upright alignment was explained by the formation of a $\mathrm{ZnO}$ wetting layer template for $\mathrm{ZnO}$ nanowire alignment; however, an explanation for the c-axis orientation of the wetting layer was not given ${ }^{17}$. Similarly, in ref. ${ }^{19}$ zinc acetate was spin-coated on the growth substrate. In that case, it was suggested that thermal decomposition of the zinc acetate produced $\mathrm{ZnO}$ colloids and nanocrystals with their (0002) planes parallel to the substrate surface. However, the question why these nuclei prefer a specific orientation has remained open.

In contrast to the reported experimental work, several theoretical papers have addressed the problem of the observed oriented growth along the c-axis in attempts to reconcile it with the expected instability of the polar faces $^{20-24}$. Wander et al. proposed that a near surface geometric relaxation, taking place gradually toward the surface over a depth of the several outmost layers along with charge transfer, stabilize the free polar surfaces of a bulk crystal ${ }^{20}$. This mechanism appears to require several crystal layers to take effect. Claeyssens et al. suggested a graphitic structure reconstruction of the $\mathrm{ZnO}$ of films thinner than 18 monolayers, over which c-oriented growth

${ }^{1}$ School of Electrical and Computer Engineering, Ben-Gurion University, Beer Sheva, 8410501, Israel. ${ }^{2}$ Department of Materials Science and Engineering, University of Michigan, 2300 Hayward, St., Ann Arbor, 48109, MI, USA. *email: shalish@bgu.ac.il 
(a)

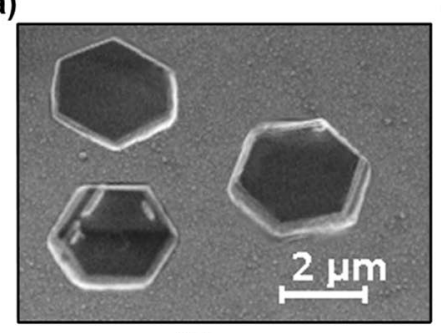

(b)

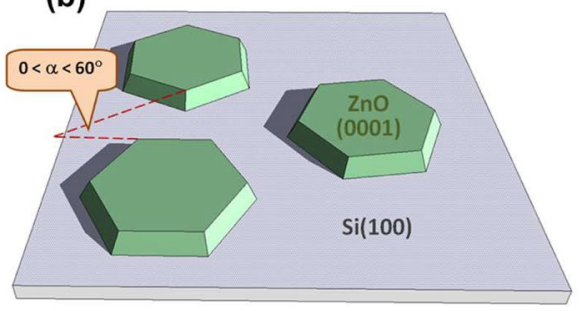

(c)

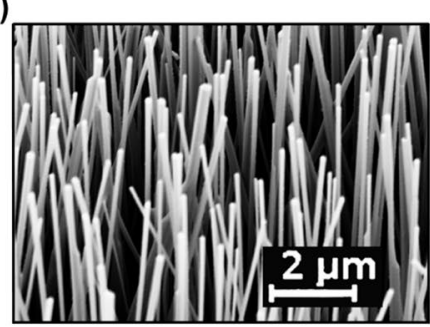

(d)

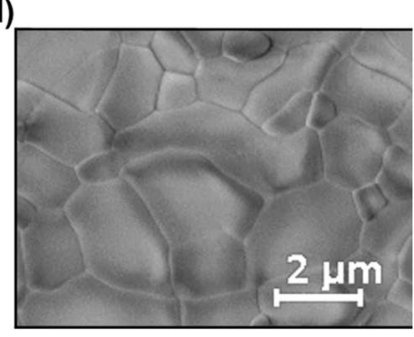

(e)

(e) electric field

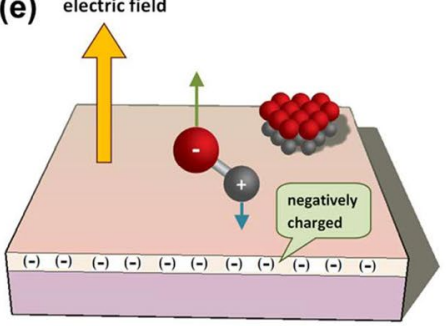

Figure 1. (a) SEM image acquired at sample-tilt of $30^{\circ}$ of hexagonally shaped mesas grown on $\mathrm{Si}(100)$ substrate, and (b) schematic cartoon showing the rotational angle difference, which confirms the lack of epitaxial relations with the substrate, despite the alignment of the c-axis perpendicular to the substrate. (c) SEM image of c-oriented $\mathrm{ZnO}$ nanowires grown on $\mathrm{Si}(100)$ substrate. (d) Polycrystalline $\mathrm{ZnO}$ film grown on a Si(100) substrate. Each individual grain is c-aligned but is rotated on the c-axis in a random angle. When they expand, they meet each other forming grain boundaries. (e) Schematic of Si substrate with negatively charged surface oxide, which exerts an electric field that aligns the polar axis of the deposited molecule along electric field lines. For $\mathrm{ZnO}$, the zinc (oxygen) face is positively (negatively) charged; thus the oxygen face points up.

should be favored ${ }^{21}$. This reconstruction was required in order to cancel the polarization charge present on the polar faces to allow the $\mathrm{ZnO}$ to grow on a substrate that was not charged. It may well be that growth of a polar crystal on a non-polar, uncharged, substrate induces a reconstruction that removes the polar nature of the single monolayer altogether.

However, as our present results seem to suggest, such reconstruction may be altogether redundant in the special case of a charged substrate. As a matter of fact, in all of these theoretical studies, no specific substrate influence was clearly considered as part of the model. However, from the thermodynamic point of view, the substrate and the deposited crystals form an interface, and the minimization of the energy of this interface provides an important driving force. If the kinetics is not too fast and the system is allowed enough time to reach equilibrium, this driving force may become important enough to derive the arrangement of the deposited crystals on the surface. Here, we propose a simple mechanism that resolves the instability problem of the polar surfaces and renders the reconstruction redundant, at least when the substrate is $\mathrm{Si}$, though the same effect may not be limited to $\mathrm{ZnO}$ on $\mathrm{Si}$.

During vapor growth of $\mathrm{ZnO}$, oxygen is an important part of the ambient. At the typically high growth temperatures, the Si substrate will oxidize faster than $\mathrm{Zn}$, due to the lower free energy of formation of $\mathrm{SiO}_{2}(\sim-727$ $\mathrm{KJ} /$ mole at $\left.1000{ }^{\circ} \mathrm{K}\right)$ compared with that of $\mathrm{ZnO}\left(\sim-256 \mathrm{KJ} / \mathrm{mole} \text { at } 1000^{\circ} \mathrm{K}\right)^{25}$. As a result, $\mathrm{SiO}_{2}$ will form immediately at the very early stages of the growth process.

The presence of oxygen at the high growth temperature is also known to induce charging in the oxide layer. Charging of the oxide during its growth has been reported already in the early days of the field effect transistor ${ }^{26}$ and is a known by-product of the oxide growth process ${ }^{27-29}$. Typically, a positive charge is induced during CVD or thermal growth, but may turn negative in plasma enhanced $\mathrm{CVD}^{30}$.

As schematically depicted in Fig. 1e, this surface charge induces an electric field perpendicular to the substrate surface. Since the polar charge on the oxygen face of $\mathrm{ZnO}$ is negative, the presence of an electric field should align the growth perpendicular to the substrate. Hence, a c-oriented growth is a reasonable outcome.

Since there are no epitaxial relations with the substrate, different individual nuclei may all have their c-axis perpendicular to the substrate but otherwise be randomly rotated. As the nuclei expand laterally, they meet one another forming a c-oriented polycrystalline film, as in Fig. 1d. Nanowire growth on such a substrate will result in upright nanowires. However, nanowires grown on different grains will have a variety of in-plane rotation angles, reflecting that of the "seed" grain.

\section{Methods}

For these studies, we utilized high-resistivity $(\sim 2000 \mathrm{Ohm} \cdot \mathrm{cm})$ boron-doped single-side polished $\mathrm{Si}(100)$ substrates. One wafer had $103 \mathrm{~nm}$-thick thermally-grown oxide. The second wafer had 88 -nm-thick plasma-enhanced-CVD-grown oxide. Charge in the surface oxide was verified using a Kelvin probe ${ }^{31}$. To form $\mathrm{ZnO}$ seed layers, $\mathrm{Zn}$-acetate was deposited and annealed at $1020^{\circ} \mathrm{C}$ for $30 \mathrm{~min}$. Due to the vanishingly small quantity of $\mathrm{ZnO}$ produced by the seeding phase, attempts to characterize it with TEM where not successful, consistent with earlier reports. The samples were placed in a quartz crucible containing a 1:1 ZnO powder: graphite 

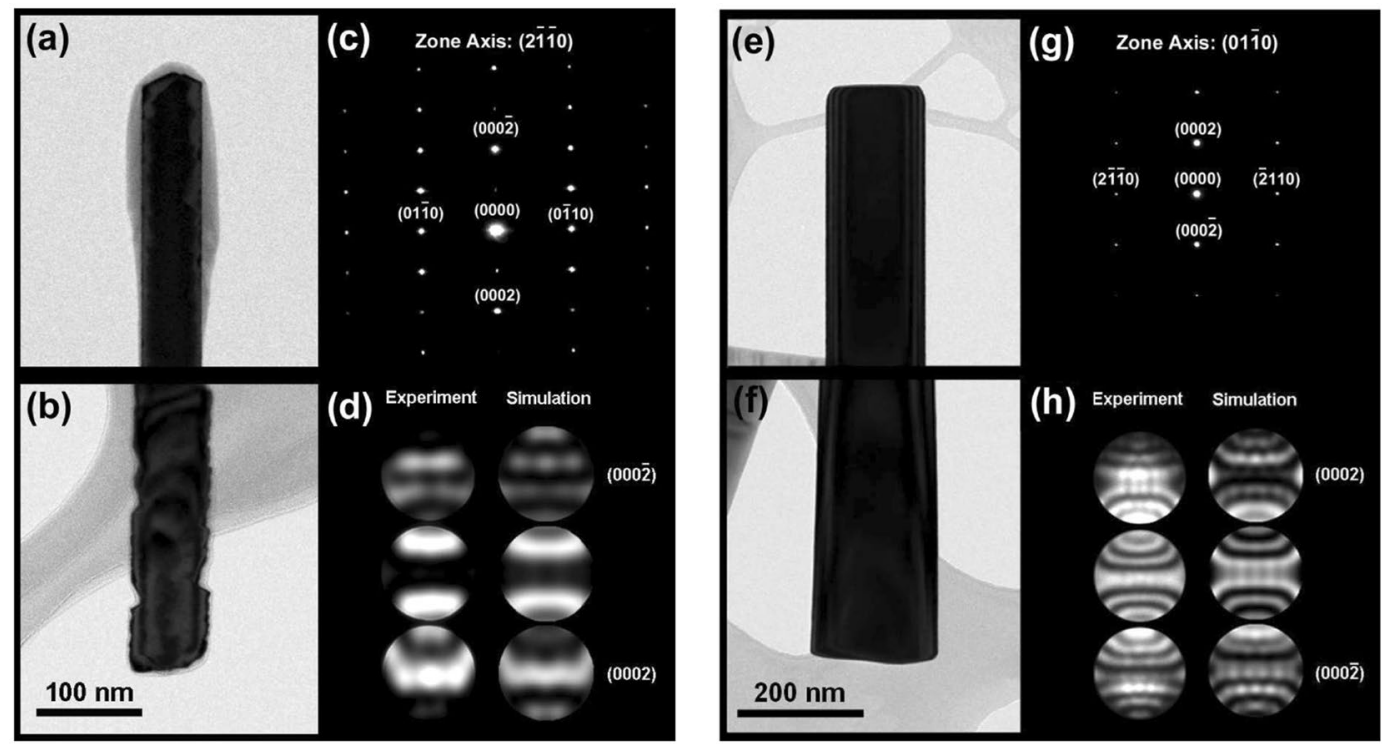

Figure 2. left - (a) TEM of the tip-end of a single CVD-grown ZnO nanowire on thermal oxide. (b) TEM image of the bottom/substrate end of the same wire. Note that the bottom of the wire is typically slightly wider with rougher surface. (c) Selective area electron diffraction (SAED), and (d) Experimental and simulated converging beam electron diffraction (CBED) corresponding to the diffraction in C acquired from the bottom end of Fig. B. The simulation corresponds to 70-nm-thick ZnO. Right - (e) TEM of the tip-end of a single CVD-grown $\mathrm{ZnO}$ nanowire on PECVD-grown oxide. (f) TEM image of the bottom/substrate end of the same wire. (g) Selective area electron diffraction (SAED) shows that the wire is oriented along the c-axis, and (h) Experimental and simulated converging beam electron diffraction (CBED) corresponding to the diffraction in (g) acquired from the bottom end of (f). The simulation corresponds to 170-nm-thick $\mathrm{ZnO}$ (the diffraction was taken slightly off the center of the wire).

mixture and introduced into the pre-heated tube furnace using a linear-motion feed-through. The $\mathrm{ZnO}$ nanowires were then grown for 5 minutes at $\sim 1020^{\circ} \mathrm{C}$ in $\mathrm{Ar} / \mathrm{O}_{2}(30 / 4 \mathrm{sccm})$ ambient. TEM imaging and electron diffraction were carried out in a JEOL JEM-2100F instrument operating at $200 \mathrm{kV}$. CBED simulation was carried out using JEMS electron microscopy simulation software ${ }^{32}$. For each oxide type, growth was repeated 3 times. For each growth, TEM/CBED imaging was carried out on $\sim 10$ nanowires.

\section{Results and Discussion}

To test our hypothesis that c-oriented $\mathrm{ZnO}$ growth on $\mathrm{SiO}_{2}$ is caused by the oxide electric charge, we consider the sign of the charge. Specifically, we consider that control of the charge sign may cause (0002) growth in the case of negative charge (electron trapping), in contrast to the commonly observed (000-2) growth on the typical hole-trapping (positively charged) thermally-grown $\mathrm{SiO}_{2}$. Therefore, we grew $\mathrm{ZnO}$ nanowires on thermally-grown, and on plasma-enhanced CVD grown, $\mathrm{SiO}_{2}$. The presence and type of surface charge was verified using a Kelvin probe. Figures 2a,b show transmission electron microscopy (TEM) images of the two ends of a CVD-grown $\mathrm{ZnO}$ nanowire grown on a positively charged thermal oxide. Diffractions were acquired at both ends of each wire to verify that no polarity inversion has taken place during the growth. The shown diffractions were obtained from the bottom/substrate end of the wire shown in Fig. 2b. The bottom part of the wire shown in Fig. $2 b$ is slightly thicker than the other end of the wire - a typical and common observation in nanowires that is useful for identification of the bottom end, especially for self-catalyzed nanowires which often lack a catalyst ball at their tip. Figure $2 \mathrm{c}$ shows the selective-area electron diffraction (SAED) pattern acquired from the bottom end of the wire, and Fig. $2 \mathrm{~d}$ shows the corresponding convergent-beam electron diffraction (CBED) pattern side by side with the simulated CBED pattern revealing a (000-2)-oriented growth direction confirming the prediction of our model, i.e., on a positively charged oxide, the O-terminated face (having positive polar charge) is the growth face, while the $\mathrm{Zn}$-terminated face (negative polar charge) faces the substrate.

The right-hand panel of Fig. 2 (Fig. 2e through $2 \mathrm{~h}$ ) follows the same structure as in the left-hand panel of Fig. 2 (Fig. 2a through 2d) showing TEM and CBED results for a CVD-grown ZnO nanowire grown on a negatively charged PECVD oxide. Comparison of the CBED pattern with simulated pattern reveals a (0002)-oriented growth direction confirming the prediction of our model for this case as well.

These diffraction results show that our $\mathrm{ZnO}$ wires grew c-oriented in both cases but flipped over their polarity when the sign of the substrate charge was changed. The wire polarity aligns parallel to the direction of the electric field. This suggests that the $\mathrm{SiO}_{2}$ substrate charge plays a pivotal role in directing the growth of the $\mathrm{ZnO}$ wires. Since electrical charging is common in insulators, and since built-in polar fields are present in many polar materials, the mechanism observed here may not be limited to the $\mathrm{SiO}_{2} \backslash \mathrm{ZnO}$ system. Charge induced during the growth of the insulating oxide substrate exerts a normal-to-substrate electric field that works to align the polar axis of the nucleating seed layer or nanowire material normal to the substrate. The polarity of the polar axis reverses with the 
sign of the substrate charge. In the case presented here, the polarity reversal and the normal-to-substrate alignment appear to be two manifestations of the same phenomenon. This mechanism appears to work as an unintentional, naturally occurring, electrophoresis on the deposited polar material. Due to their built-in electric field and dipolar nature, wurtzite materials possess an inner mechanism that affects their self-assembly into crystals ${ }^{33}$.

Several studies on thin films of polycrystalline $\mathrm{Cu}$ showed preferred crystallographic orientation (i.e. crystallographic texture) when grown on amorphous $\mathrm{SiO}_{2}$ substrates, although epitaxy was not possible ${ }^{34,35}$. In these cases, $\mathrm{Cu}$, which has a face-centered-cubic structure, tends to prefer the (111) orientation. Annealing of these films close to their melting points often increases the (111) texture. This specific orientation of the copper on the $\mathrm{SiO}_{2}$ surface appears to minimize the surface free energy. In general, the surface free energy $\overline{\mathrm{f}}$ is given by ${ }^{36}$

$$
\overline{\bar{f}}=\gamma+\sum_{i} \mu_{i} \Gamma_{\mathbf{i}}
$$

where $\gamma$ is the surface tension, $\boldsymbol{\mu}_{\boldsymbol{i}}$ is the chemical potential of component $\boldsymbol{i}$, and $\boldsymbol{\Gamma}_{\mathrm{i}}$ is the adsorption of component $i$. Since, in the $\mathrm{Cu}$ case, the grown crystal is a metal, one may safely ignore any possible influence of an electric field induced by electric charges in the substrate and consider the differences in the surface chemical potential alone. However, for polar materials, the effect of an electric field cannot be ignored, and the added electrical potential component is expressed in a replacement of the chemical potentials, $\boldsymbol{\mu}_{i}$, by electrochemical potentials, $\widetilde{\mu}_{i}$, given by the Guggenheim relationship ${ }^{36,37}$.

$$
\widetilde{\mu}_{i}=\mu_{i}+q z_{i} \varphi_{i}
$$

where $\mathbf{q}$ is the elementary charge, $z_{i}$ is the ion charge number, and $\varphi_{i}$ is the electrical potential. The contribution of the electrical potential to the interfacial energy depends both on the electric field emanating from the substrate and on the built-in polar field in the unit cell of the polar material. Hence, the strength of this effect may depend on the strength of the built-in polar electric field.

Our experiment shows a clear correlation of the $\mathrm{ZnO}$ growth polarity with the sign of the substrate charge. Previous studies have suggested that the chemical potential on the $\mathrm{Zn}$-face of $\mathrm{ZnO}$ is quite different from that on the $\mathrm{O}$-face ${ }^{38}$. This would imply that in the absence of electrical potentials, the $\mathrm{ZnO}$ system would prefer one growth polarity over the other, due to this difference in the chemical potentials. Indeed, growth of $\mathrm{ZnO}$ has been reported to prefer the (0002) polar orientation over $(000-2)^{39,40}$. In our case of an intentionally charged substrate, we observed growth in both polarities depending on the polarity of substrate charge. This suggests that the effect of the electrical potentials on the interface energy of the $\mathrm{ZnO} / \mathrm{SiO}_{2}$ system prevails over that of the chemical potentials. Thus, in spite of the difference in chemical potentials between the oxygen and the zinc-terminated polar faces of $\mathrm{ZnO}$, the surface energy minimization achieved by the electrical alignment of the deposited polar molecules is apparently much greater than that which could be achieved by chemical potentials alone.

As previously discussed, several theoretical studies have considered the expected instability of the polar faces of $\mathrm{ZnO}$ and suggested that reconstruction in inevitable to explain their observed stability ${ }^{20-24}$. These studies have not considered the possible effect of specific substrates. Our results suggest that substrate charge may as well stabilize the polar face. Thus, the influence of the substrate electric field is likely to provide additional control over the growth of polar materials.

The substrate electric field applies torque to the $\mathrm{ZnO}$ dipole. The potential energy of the dipole is minimized only when the dipole is aligned parallel to the electric field. Since the electric field is aligned perpendicular to the substrate, the polar axis of the $\mathrm{ZnO}$ wires also aligns perpendicular to the substrate, resulting in the observed normal-to-surface growth.

To facilitate the polar alignment, it seems necessary to grow the nanowires in a two-step process. In the first step, or the nucleation step, it is necessary to limit the amount of the nucleating material, to enable the formation of a seed layer, wherein the seeds are aligned. In the second step, epitaxial growth of nanowires may take place on the seed layer. If the first step is bypassed, the typically high nanowire growth rate disturbs the alignment. High growth rate may sometimes disturb alignment even at the presence of epitaxial guidance, let alone in its absence, while low growth rate is more favorable even for facilitating epitaxy ${ }^{41}$. In other words, the kinetics may be too fast, and the system is not allowed enough time to reach equilibrium. In contrast, when using the $\mathrm{Zn}$-acetate method, the quantity of $\mathrm{Zn}$ that is available for nucleation appears to be small enough to allow optimal alignment of the particles that may then serve as seeds for epitaxial growth of $\mathrm{ZnO}$ nanowires.

Effects of oxide substrate on polarity of nanowires has also been reported in other material systems. GaN nanowires grown on $\mathrm{Si}(111)$ showed $\mathrm{N}$-polarity, but the polarity was flipped over when oxynitrides of $\mathrm{Al}$ and $\mathrm{Ga}$ were used as an intermediate layer ${ }^{42}$.

Although the effect we report is more easily observed in nanowires, it may not be limited to nanowires and may also affect the growth of films. For example, lateral overgrowth of $\mathrm{GaN}_{\text {often }}$ uses a mask of $\mathrm{SiO}_{2}$. The GaN grows up through openings in the mask and then grows laterally over the masked areas. In some cases, domains of reversed polarity have been observed in areas on the $\mathrm{SiO}_{2}$ to which the $\mathrm{GaN}$ as grown laterally, though this polarity reversal has often been attributed to other phenomena ${ }^{43,44}$. Polarity inversion was also observed in liquid-phase epitaxy of AIN on nitrided sapphire and was shown to relate to the partial pressure of oxygen during the growth, possibly forming an oxide interface ${ }^{45}$.

Finally, wurtzite is by no means the only polar crystal structure. There are several other forms known to be polar. For example, among the common compound semiconducting materials, the zincblende structure is very common. The polarity of zincblende crystals is typically much weaker than that in wurtzite. It seems likely that for this reason, the phenomenon we discuss is more readily observed in $\mathrm{ZnO}$ than, e.g., in III-V compounds, where substrate alignment of nanowires is commonly explained by epitaxy ${ }^{46}$. 


\section{Conclusion}

The observed correlation of polar direction of $\mathrm{ZnO}$ nanowires with the sign of the $\mathrm{SiO}_{2}$ substrate charge suggests that the alignment of the $\mathrm{ZnO}$ polar axis perpendicular to the substrate is the effect of the electric field emanating from the substrate. The proposed mechanism may not be limited to the $\mathrm{ZnO} / \mathrm{Si}$ system. It sets forth a new concept, according to which substrate charge may be exploited to affect the growth direction of polar semiconductors.

Received: 13 December 2019; Accepted: 1 April 2020;

Published online: 16 April 2020

\section{References}

1. Özgür, Ü. et al. A comprehensive review of ZnO materials and devices. J. Appl. Phys. 98, 041301, https://doi.org/10.1063/1.1992666 (2005).

2. Polman, A., Knight, M., Garnett, E. C., Ehrler, B. \& Sinke, W. C. Photovoltaic materials: Present efficiencies and future challenges. Science 352, 307, https://doi.org/10.1126/science.aad4424 (2016).

3. Zhao, H., Hu, J., Chen, S., Xie, Q. \& He, J. High Nonlinearity and High Voltage Gradient ZnO Varistor Ceramics Tailored by Combining $\mathrm{Ga}_{2} \mathrm{O}_{3}, \mathrm{Al}_{2} \mathrm{O}_{3}$, and $\mathrm{Y}_{2} \mathrm{O}_{3}$ Dopants. J. Am. Ceram. Soc. 99, 769, https://doi.org/10.1111/jace.14110 (2016).

4. Alenezi, M. R., Henley, S. J. \& Silva, S. R. P. On-chip Fabrication of High Performance Nanostructured ZnO UV Detectors. Sci. Rep. 5, 8516, https://doi.org/10.1038/srep08516 (2015).

5. Drobek, M. et al. MOF-Based Membrane Encapsulated ZnO Nanowires for Enhanced Gas Sensor Selectivity. ACS Appl. Mater. Interfaces 8, 8323-8328, https://doi.org/10.1021/acsami.5b12062 (2016).

6. Ong, C. B., Ng, L. Y. \& Mohammad, A. W. A review of ZnO nanoparticles as solar photocatalysts. Ren. Sustain. Ener. Rev. 81, 536-551, https://doi.org/10.1016/j.rser.2017.08.020 (2018).

7. Shih, C.-C. et al. High Performance Transparent Transistor Memory Devices Using Nano-Floating Gate of Polymer/ZnO Nanocomposites. Sci. Rep. 6, 20129, https://doi.org/10.1038/srep20129 (2016).

8. Lu, J. et al. Plasmon-enhanced Electrically Light-emitting from ZnO Nanorod Arrays/p-GaN Heterostructure Devices. Sci. Rep. 6, 25645, https://doi.org/10.1038/srep25645 (2016).

9. Kerr Barnes, B. \& Das, K. S. Resistance Switching and Memristive Hysteresis in Visible-Light-Activated Adsorbed ZnO. Thin Films. Sci. Rep. 8, 2184, https://doi.org/10.1038/s41598-018-20598-5 (2018).

10. Wahab, H. A. et al. Zinc oxide nano-rods based glucose biosensor devices fabrication. Results in Physics 9, 809-814, https://doi. org/10.1016/j.rinp.2018.02.077 (2018).

11. Zhu, L. et al. Piezotronic Effect on Rashba Spin-Orbit Coupling in a ZnO/P3HT Nanowire Array Structure. ACS Nano 12, 1811-1820, https://doi.org/10.1021/acsnano.7b08618 (2018).

12. Aras, M. \& Kılıç, Ç. Electrical tuning of spin splitting in Bi-doped ZnO nanowires. Phys. Rev. B 97, 035405, https://doi.org/10.1103/ PhysRevB.97.035405 (2018).

13. Zhou, Q., Wen, J. Z., Zhao, P. \& Anderson, W. A. Synthesis of Vertically-Aligned Zinc Oxide Nanowires and Their Application as a Photocatalyst. Nanomaterials 7, 9, https://doi.org/10.3390/nano7010009 (2017).

14. Park, W., Kim, D. H., Jung, S. \& Yi, G.-C. Metalorganic vapor-phase epitaxial growth of vertically well-aligned ZnO nanorods. Appl. Phys. Lett. 80, 4232, https://doi.org/10.1063/1.1482800 (2002).

15. J. Wu, S.C. Liu. Low-Temperature Growth of Well-Aligned ZnO Nanorods by Chemical Vapor Deposition. Adv. Mater. 14, 215 10.1002/1521-4095(20020205)14:3<215::AID-ADMA215>3.0.CO;2-J (2002).

16. Li, Q. et al. Fabrication of ZnO Nanorods and Nanotubes in Aqueous Solutions. Chem. Mater. 17, 1001-1006, https://doi. org/10.1021/cm048144q (2005).

17. Zhang, H., Sun, X., Wang, R. \& Yu, D. Growth and formation mechanism of c-oriented ZnO nanorod arrays deposited on glass. Cryst. Growth 269, 464-471, https://doi.org/10.1016/j.jcrysgro.2004.05.078 (2004).

18. Ma, T., Guo, M., Zhang, M., Zhang, Y. \& Wang, X. Density-controlled hydrothermal growth of well-aligned ZnO nanorod arrays. Nanotechnology 18, 035605, https://doi.org/10.1088/0957-4484/18/3/035605 (2007).

19. Greene, L. E. et al. General Route to Vertical ZnO Nanowire Arrays Using Textured ZnO Seeds. Nano Lett. 5, 1231-1236, https://doi. org/10.1021/nl050788p (2005).

20. Wander, A. et al. Stability of Polar Oxide Surfaces. Phys. Rev. Lett. 86, 3811, https://doi.org/10.1103/PhysRevLett.86.3811 (2001).

21. Claeyssens, F. et al. Growth of ZnO thin films-experiment and theory. J. Mater. Chem. 15, 139-148, https://doi.org/10.1039/ B414111C (2005)

22. Freeman, C. L., Claeyssens, F., Allan, N. L. \& Harding, J. H. Graphitic Nanofilms as Precursors to Wurtzite Films: Theory. Phys. Rev. Lett 96, 066102, https://doi.org/10.1103/PhysRevLett.96.066102 (2006).

23. Noguera, C. \& Goniakowski, J. Polarity in oxide ultrathin films. J. Phys.: Condens. Matter 20, 264003, https://doi.org/10.1088/0953$8984 / 20 / 26 / 264003$ (2008).

24. Mora-Fonz, D. et al. Why Are Polar Surfaces of ZnO Stable? Chem. Mater. 29, 5306-5320, https://doi.org/10.1021/acs. chemmater.7b01487 (2017).

25. W. F. Gale \& T. C. Totemeier, Eds. Smithells Metals Reference Book, 8th ed. Amsterdam: Elsevier. ISBN 0750675098. p. 8-26 2004.

26. Atalla, M. M., Tannenbaum, E. \& Scheibner, E. J. Stabilization of Silicon Surfaces by Thermally Grown Oxides. Bell Sys. Tech. J. 38, 749, https://doi.org/10.1002/j.1538-7305.1959.tb03907.x (1959).

27. Deal, B. E., Sklar, M., Grove, A. S. \& Snow, E. H. Characteristics of the Surface-State Charge (Qss) of Thermally Oxidized Silicon. J. Electrochem. Soc. 114, 266-274, https://doi.org/10.1149/1.2426565 (1967).

28. Deal, B. E. The Current Understanding of Charges in the Thermally Oxidized Silicon Structure. J. Electrochem. Soc. 121, 198C-205C, https://doi.org/10.1149/1.2402380 (1974).

29. Boogaard, A., Kovalgin, A. Y. \& Wolters, R. A. M. Net Negative Charge in low-temperature SiO2 gate dielectric layers. Microelectron. Eng. 86, 1707-1710, https://doi.org/10.1016/j.mee.2009.03.124 (2009).

30. Boogaard, A., Kovalgin, A. Y. \& Wolters, R. A. M. Negative Charge in Plasma Oxidized SiO2 Layers. ECS Transactions 35, 259-272, https://doi.org/10.1149/1.3572288 (2011).

31. For details of the technique, see D. K. Schroder. Semiconductor Material and Device Characterization, 3rd ed. New Jersey: Wiley. ISBN: 978-0-471-73906-7. p. 538. 2006.

32. P. Stadelmann. JEMS, electron microscopy software, java version. http://cimewww.epfl.ch/people/stadelmann/jemsWebSite/jems. html 2004.

33. Turkulets, Y. \& Shalish, I. Polar-charge-induced self-assembly: Electric effect that causes nonisotropic nanorod growth in wurtzite semiconductors. Phys. Rev. Mater. 3, 033403, https://doi.org/10.1103/PhysRevMaterials.3.03340 (2019).

34. Bagalagel, S. \& Shirokoff, J. Effect of annealing on preferred orientations in the $\mathrm{Cu} / \mathrm{SiO} 2$ and Cu/SiO2/Si(100) interfaces. Mater. Sci. Eng. A 479, 112-116, https://doi.org/10.1016/j.msea.2007.06.032 (2008).

35. Perez-Prado, M. T. \& Vlassak, J. J. Microstructural evolution in electroplated Cu thin films. Scripta Materialia 47, 817-823, https:// doi.org/10.1016/S1359-6462(02)00308-1 (2002).

36. Rusanov, A. I. Thermodynamics of solid surfaces. Surf. Sci. Rep. 23, 173-247, https://doi.org/10.1016/0167-5729(95)00007-0 (1996). 
37. Guggenheim, E. A. The Conceptions of Electrical Potential Difference between Two Phases and the Individual Activities of Ions. J. Phys. Chem. 33, 842-849, https://doi.org/10.1021/j150300a003 (1929).

38. Tang, C., Spencer, M. J. S. \& Bernard, A. S. Activity of ZnO polar surfaces: an insight from surface energies. Phys. Chem. Chem. Phys. 16, 22139-22144, https://doi.org/10.1039/C4CP03221G (2014).

39. Baxter, J., Wu, F. \& Aydil, E. Growth mechanism and characterization of zinc oxide hexagonal columns. Appl. Phys. Lett. 83, 3797, https://doi.org/10.1063/1.1624467 (2003).

40. Wang, Z., Kong, X. \& Zuo, J. Induced Growth of Asymmetric Nanocantilever Arrays on Polar Surfaces. Phys. Rev. Lett. 91, 185502, https://doi.org/10.1103/PhysRevLett.91.185502 (2003).

41. Zuniga-Perez, J. et al. Ordered growth of tilted $\mathrm{ZnO}$ nanowires: morphological, structural and optical characterization. Nanotechnol. 18, 195303, https://doi.org/10.1088/0957-4484/18/19/195303 (2007).

42. Concordel, A. et al. Polarity conversion of GaN nanowires grown by plasma-assisted molecular beam epitaxy. Appl. Phys. Lett. 114, 172101, https://doi.org/10.1063/1.5094627 (2019).

43. Lee, H., Jang, D., Kim, D. \& Kim, C. Non-edge-triggered inversion from Ga polarity to N polarity of c-GaN domains on an SiO2 mask during epitaxial lateral overgrowth. J. Appl. Cryst. 52, 532, https://doi.org/10.1107/S1600576719003662 (2019).

44. Song, J., Yuan, G., Xiong, K., Leung, B. \& Han, J. Epitaxial Lateral Overgrowth of Nitrogen-Polar (0001) GaN by Metalorganic Chemical Vapor Deposition. Cryst. Growth Des. 14, 2510, https://doi.org/10.1021/cg500229r (2014).

45. Adachi, M. et al. Polarity inversion and growth mechanism of AlN layer grown on nitride sapphire substrate using Ga-Al liquidphase epitaxyPhys. Status Solidi B 252, 743, https://doi.org/10.1002/pssb.201451426 (2015).

46. MårtenssonC., T. et al. Epitaxial III-V Nanowires on Silicon. Nano Letters 4, 1987 (2004).

\section{Acknowledgements}

We acknowledge the Ilse Katz Institute for Nanoscale Science \& Technology at Ben Gurion University and Michigan Center for Materials Characterization for use of the instruments and staff assistance. We gratefully acknowledge the support of BSF grant \#2015700 and NSF grant \# ECCS-1610362.

\section{Author contributions}

I.S. conceived the study and designed the experiments. A.R.A., Y.T. and D.G. carried out the experiments and data analysis. I.S. and R.S.G. co-wrote the paper. All authors contributed to the final version of the paper.

\section{Competing interests}

The authors declare no competing interests.

\section{Additional information}

Supplementary information is available for this paper at https://doi.org/10.1038/s41598-020-63500-y.

Correspondence and requests for materials should be addressed to I.S.

Reprints and permissions information is available at www.nature.com/reprints.

Publisher's note Springer Nature remains neutral with regard to jurisdictional claims in published maps and institutional affiliations.

(c) (i) Open Access This article is licensed under a Creative Commons Attribution 4.0 International License, which permits use, sharing, adaptation, distribution and reproduction in any medium or format, as long as you give appropriate credit to the original author(s) and the source, provide a link to the Creative Commons license, and indicate if changes were made. The images or other third party material in this article are included in the article's Creative Commons license, unless indicated otherwise in a credit line to the material. If material is not included in the article's Creative Commons license and your intended use is not permitted by statutory regulation or exceeds the permitted use, you will need to obtain permission directly from the copyright holder. To view a copy of this license, visit http://creativecommons.org/licenses/by/4.0/.

(C) The Author(s) 2020 